\title{
Cores of $1 / 2<110>-$ type dislocations in the CrMnFeCoNi high-entropy alloy investigated by STEM, the center of symmetry and the Nye tensor mapping techniques
}

Milan Heczko $^{1}$, Veronika Mazánová ${ }^{1}$, Roman Gröger $^{2}$, Tomáš Záležák ${ }^{2}$, Mohammad S. Hooshmand ${ }^{3}$, Easo George ${ }^{4}$, Michael Mills ${ }^{1}$ and Antonín Dlouhý ${ }^{2}$

${ }^{1}$ The Ohio State University, Columbus, Ohio, United States, ${ }^{2}$ Institute of Physics of Materials, Czech Academy of Sciences, Brno, Jihomoravskykraj, Czech Republic, ${ }^{3}$ University of California Berkeley, Berkeley, California, United States, ${ }^{4}$ Oak Ridge National Laboratory, Oak Ridge, Tennessee, United States

The strength of high-temperature materials generally stems from two- or multi-phase microstructures in which the matrix phase is a complex solid solution containing many elements. It is thus not surprising that the increased attention is focused on the mechanical properties of recently developed multi-principal element alloys (MPEA), including the single-phase CrMnFeCoNi alloy with the face-centered cubic (FCC) lattice $[1,2]$. Due to their high configurational entropy these equiatomic solid solutions were expected to possess improved thermodynamic stability and remain in the solid solution state, particularly at high temperatures where the entropy term dominates the Gibbs free energy [3]. However, several studies have now demonstrated that even the Cantor alloy ( $\mathrm{CrMnFeCoNi})$, which was once thought to be the exemplar of a stable solid solution, decomposes into a multiphase state at elevated temperatures in the range 723 - $973 \mathrm{~K}$. The role of dislocations in this process needs further exploration because their cores represent paths of high diffusivity. Additionally, the possibility of elemental enrichment may fundamentally alter the motion of dislocations during high temperature deformation. Atomic resolution characterization of the $1 / 2<110>$-type dislocations with particular attention given to the core structure and its chemical composition is therefore very important [4].

To that end, CrMnFeCoNi samples were first deformed in tension at room temperature to plastic strains of 0.2 and $2.3 \%$, and subsequently annealed at $973 \mathrm{~K}$ for 800 hours. The pre-strains activated planar slip of $1 / 2<110>$-type dislocations on $\{111\}$-type glide planes (Fig. 1a). Interactions of this planar slip with special $\Sigma 3$ grain boundaries formed a large number of dislocation segments with a $<110>$-type crystallographic orientation suitable for a credible end-on analysis of dislocation cores in the highresolution scanning transmission electron microscope (HR-STEM), see Fig. 1b. STEM foils tilted into specific low index crystallographic zones of the $<110>$-type were investigated in a high-angle annular dark-field (HAADF) regime in an image-corrected and monochromated Thermo Fisher Scientific (TFS)/FEI Titan-Themis 60-300 kV S/TEM. The corresponding atomic resolution images together with the high spatial resolution chemical data from a Super-X EDS detector provided detailed information on the atomic structure and chemical composition of the dislocation cores. The data were collected and processed using the TFS Velox software. The atomic resolution STEM images were corrected for possible drift and scanning beam distortions using the drift corrected frame integration (DCFI) function of Velox.[4]

Maps of dislocation cores were generated by the center of symmetry (COS) and the Nye tensor techniques [4]. The COS analysis determines the degree of centro-symmetry for each atomic column in the experimental HAADF-STEM image and thus identifies faults in the stacking sequence (see Fig. 1c) [5]. Centroids of the atomic columns are determined by cross correlating the images with Gaussian peaks with well-defined maxima. The Nye tensor component distributions are computed by a modification of the 
method proposed by Hartley and Mishin [6] for 3D crystals that made it applicable to 2D HAADF-STEM images. The 3D lattice is projected onto the plane of view, perpendicular to the electron beam and the dislocation line. The projected lattice is treated as a 2D lattice with newly defined nearest neighbor vectors. The 2D Nye analysis does not provide any information about the screw component of the Burgers vector due to a lack of displacement data parallel to the dislocation line. The ability of the COS to detect the entire stacking fault is complementary to the Nye tensor analysis which, on the other hand, provides better information on the positions of the Shockley partials (see Fig. 1d) [4, 7].

The current study revealed that the cores of $1 / 2<110>$ dislocations pushed against the $\Sigma 3$ grain boundaries are close to the compact configuration. The width of stacking fault ribbons depends on (i) the sum of the pushing force due to dislocation-dislocation interactions and the superposed applied stress and/or (ii) the local chemical arrangements along the stacking fault plane and the related variations in the local stacking fault energy. Within the sensitivity of the Super-X EDS mapping, there was no concentration gradient detected which would confirm an enrichment of dislocation cores by any elemental constituent of the alloy after the annealing at $973 \mathrm{~K}$ for 800 hours. Under these conditions, the elastic interactions between the stress fields of isolated individual dislocations and the elemental constituents of the CrMnFeCoNi alloy are not sufficient to drive any detectable segregation to dislocations. [4, 8]

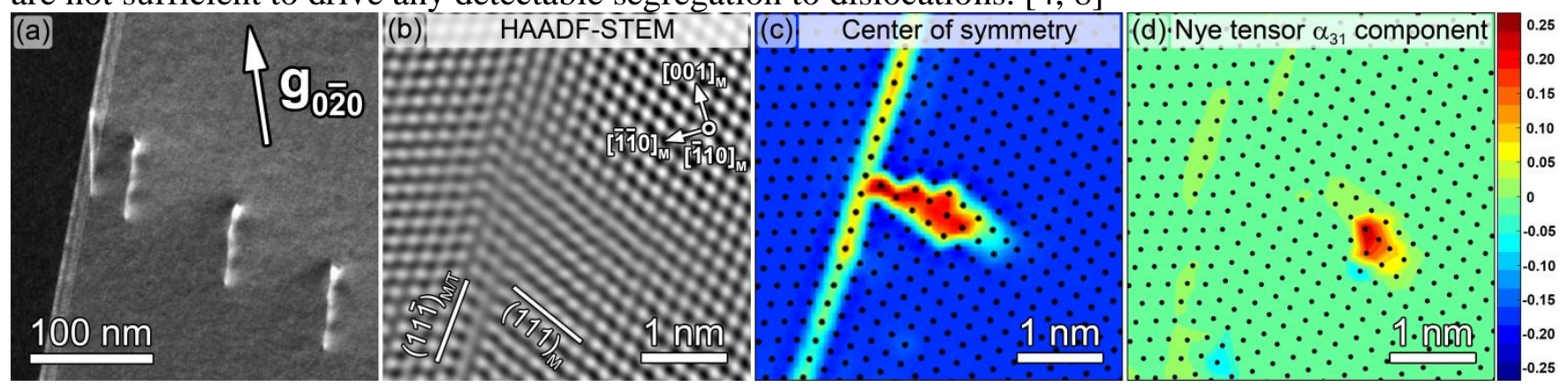

Figure 1. Figure 1. (a) Planar dislocation slip with the screw dislocation at the head of the pile-up oriented parallel to the crystallographic direction. (b) HAADF-STEM showing the core of a $1 / 2$ dislocation in the equiatomicCrMnFeCoNi alloy after tensile pre-deformation and annealing at $973 \mathrm{~K}$ for 800 hours. Corresponding (c) COS and (d) $\alpha 31$ Nye tensor map.[4]

\section{References}

[1] Cantor et al., Mat. Sci. Eng. A, 375-377 (2004), pp. 213-218.

[2] Otto et al., Acta Mater., 61 (2013), pp. 5743-5755.

[3] Yeh et al., Adv. Eng. Mater., 6 (2004), pp. 299-303.

[4] Heczko et al., Acta Mater., 208 (2021), 116719.

[5] Vorontsov et al., Acta Mater., 60 (2012), pp. 4866-4878.

[6] Hartley and Mishin, Acta Mater., 53 (2005), pp. 1313-1321.

[7] Smith et al., Acta Mater., 110 (2016), pp. 352-363.

[8] VM, RG, TZ and AD acknowledge financial support from the CSF under the contract no. 14-22834S. EPG is supported by the U.S. Department of Energy, Office of Science, Basic Energy Sciences, Materials Sciences and Engineering Division. MJM, MH and VM acknowledge financial support from the NSF under the contract No. \#DMR-1905748. The authors acknowledge support from the Thermo Fisher Scientific \& Czechoslovak Microscopy Society fellowship awarded to MH. 